\title{
Uso da cutina na estimativa da digestibilidade aparente de dietas para equinos
}

\author{
[Use of cutin for determining apparent digestibility of diets for horses] \\ R.F. Siqueira ${ }^{1}$, R.C. Gomes ${ }^{2}$, P.H.M. Rodrigues ${ }^{3}$, R.S. Fukushima ${ }^{3}$, \\ C.L.F. Lorenzo ${ }^{4}$, A.A.O. Gobesso ${ }^{3}$ \\ ${ }^{1}$ Aluna de pós-graduação - FMVZ-USP - São Paulo, SP \\ ${ }^{2}$ Aluno de pós-graduação - FZEA-USP - Pirassununga, SP \\ ${ }^{3}$ Faculdade de Medicina Veterinária e Zootecnia - USP - São Paulo, SP \\ ${ }^{4}$ Zootecnista autônomo
}

\begin{abstract}
RESUMO
Avaliaram-se a acurácia, a precisão e a robustez dos indicadores cutina, lignina em detergente ácido, óxido crômico e coleta total de fezes na estimativa da digestibilidade aparente da matéria orgânica de dietas para equinos. Para tal, foram utilizados quatro equinos machos, com idade aproximada de 10 meses e média de peso de $197 \mathrm{~kg}(170 \mathrm{a} 216 \mathrm{~kg})$. O experimento foi realizado em quatro períodos, com duração de 11 dias cada, sendo os oito primeiros usados para adaptação às dietas e os três subsequentes, para colheita de material. O delineamento experimental foi em quadrado latino 4X4. A ponderação dos coeficientes de digestibilidade da matéria orgânica pelos indicadores foi efetuada por meio do viés. A acurácia e a precisão foram determinadas pela comparação entre os dados preditos e observados, e a robustez pela comparação dos vieses com outros fatores estudados. A cutina não se mostrou eficiente como indicador interno, pois superestimou a digestibilidade aparente da matéria orgânica e resultou em menor acurácia e precisão. $\mathrm{O}$ oxido crômico apresentou baixa recuperação fecal e subestimou a digestibilidade aparente da matéria orgânica, embora tenha sido o mais preciso. A lignina em detergente ácido foi o indicador que obteve a melhor recuperação fecal e foi o mais acurado, portanto, o indicador mais eficiente.
\end{abstract}

Palavras-chave: equino, digestibilidade, indicadores, acurácia, precisão

\begin{abstract}
The accuracy, precision, and robustness of the cutin, acid detergent lignin (ADL), chromic oxide, and total feces collection to estimate the apparent digestibility of the organic matter of diets for equines were evaluated. For such, four male horses were used. They averaged 10 month-old and $197 \mathrm{~kg}$ (170 to $216 \mathrm{~kg}$ ). The experiment was carried out in four periods with duration of eleven days each, being the first eight for adaptation to the diets and the three subsequent to obtain the results. The experimental design was a $4 x 4$ latin square. The balance of the coefficients of digestibility of the organic matter for the markers was made by means of the bias. The accuracy and the precision were determined by the comparison of the predicted data with the observed ones, and the robustness by the comparison of the bias with other studied factors. The cutin did not show efficient as an internal marker, therefore it overestimated the apparent digestibility of the organic matter and showed to be less accurate and precise. The chromic oxide presented low fecal recovery and underestimated the apparent digestibility of the organic matter, even though it was more precise. The acid detergent lignin was the marker that got the best fecal recovery and was the most accurate, therefore, the most efficient marker.
\end{abstract}

Keywords: horse, digestibility, markers, accuracy, precision

Recebido em 18 de fevereiro de 2009

Aceito em 8 de outubro de 2009

E-mail: refarinelli@yahoo.com.br 


\section{INTRODUÇÃO}

A digestibilidade dos alimentos pode ser determinada in vivo pelo método de coleta total de fezes ou estimada por uso de indicadores. A recuperação de frações indigeríveis do alimento é a base para os indicadores internos, que são utilizados em estudos nos quais são necessárias estimativas de digestibilidade (van Soest, 1994). $\mathrm{O}$ erro de amostragem pode ser reduzido se um componente indigestível de alta porcentagem na matéria seca puder ser encontrado. Nesse sentido, tem sido sugerido que as frações fibrosas indigeríveis do alimento sejam utilizadas com esse propósito (Lippke et al., 1986).

Pereira et al. (1995) e Araujo et al. (2003) e Silva et al. (2006) utilizaram vários indicadores, como cinzas insolúveis em ácido, cinzas insolúveis em detergente ácido, lignina em detergente ácido, fibra em detergente neutro indigestível, óxido crômico e obtiveram resultados controversos e dependentes da dieta utilizada.

A cutina é um polímero de ácidos graxos esterificados ligados nas partes aéreas (folhas, frutos e talos) de algumas plantas. Silva et al. (2006) utilizaram-na como indicador interno para o cálculo do coeficiente de digestibilidade dos componentes nutricionais de rações para bovinos, e os resultados indicaram o seu potencial para esse fim.

Os objetivos deste trabalho foram testar o uso da cutina como possível indicador interno e avaliar a acurácia, a precisão e a robustez dos indicadores cutina, lignina em detergente ácido e óxido crômico, em comparação à coleta total de fezes, na estimativa da digestibilidade aparente da matéria orgânica de dietas em equinos.

\section{MATERIAL E MÉTODOS}

Foram utilizados quatro equinos machos, filhos do mesmo garanhão, sem raça definida, com idade aproximada de 10 meses e média de peso de $197 \mathrm{~kg}$ (170 a $216 \mathrm{~kg})$, alojados em baias individuais. $\mathrm{O}$ experimento foi realizado em quatro períodos com duração de 11 dias cada, sendo os oito primeiros usados para adaptação às dietas e os três subsequentes, para coleta de material (Araújo et al., 2003).

Os animais foram alimentados duas vezes ao dia, sendo a ração total (feno + concentrado) dividida em partes iguais entre os dois horários (7 e 19 horas). A dieta foi fornecida em 2,5\% do peso vivo com base na matéria seca, sendo composta por $40 \%$ de concentrado e $60 \%$ de volumoso, de acordo com a orientação do NRC (Nutrient..., 1989) para essa categoria.

Seguindo o delineamento em quadrado latino $4 \mathrm{x}$ 4, os animais foram distribuídos aleatoriamente em um dos seguintes tratamentos: AC - dieta contendo feno de alfafa disposto em cubos, AR dieta contendo feno de alfafa disposto em ramas, $\mathrm{AC}+\mathrm{O}$ - dieta com feno de alfafa em cubos adicionado de óleo de soja refinado e AR + O dieta com feno de alfafa em ramas adicionado de óleo de soja refinado.

A coleta total de fezes foi realizada do nono ao $11^{\circ}$ dia experimental (três períodos de quatro horas), com os animais mantidos nas baias, sem cama. As fezes foram recolhidas do chão, pesadas e identificadas individualmente. Das fezes, após homogeneização, foram retiradas frações correspondentes a $10 \%$ do total, acondicionadas em sacos plásticos e congeladas a $-20^{\circ} \mathrm{C}$ para posteriores análises laboratoriais. Em todos os dias de cada período de coleta, junto às duas refeições, foram adicionados $10 \mathrm{~g}$ de óxido crômico misturado a pequenas quantidades de concentrado, totalizando $20 \mathrm{~g}$ do indicador, por dia. A quantificação do cromo nas fezes deuse de acordo com a metodologia proposta por Uden et al. (1980), utilizando-se espectrofotometria de absorção atômica ${ }^{1}$.

$\mathrm{Na}$ Tab. 1, encontra-se o consumo dos indicadores em função dos tratamentos utilizados.

A determinação da concentração de lignina em detergente ácido (LDA) nos alimentos e nas fezes foi realizada conforme metodologia descrita por Van Soest (1964), citada por Silva (1990). As análises do conteúdo de cutina nos ingredientes e fezes foram realizadas a partir da LDA, (Fukushima, 2007; FMVZ-USP, SP; comunicação pessoal). Os indicadores cutina, LDA e óxido crômico para a estimativa da digestibilidade aparente da matéria orgânica (DAMO) foram avaliados quanto à acurácia, precisão e robustez, em relação à coleta total de fezes.

${ }^{1}$ Aparelho Hitachi, modelo Z-8200, Mitorika, Ibaraki, Japão. 
Uso da cutina na estimativa da digestibilidade...

Tabela 1. Consumo de matéria seca diário da dieta, concentração dos indicadores na dieta e ingestão diária dos indicadores em função dos tratamentos usados para equinos

\begin{tabular}{llcccc}
\hline Consumo & & $\mathrm{AC}$ & $\mathrm{AC}+\mathrm{O}$ & $\mathrm{AR}$ & $\mathrm{AR}+\mathrm{O}$ \\
\hline Dieta & $\mathrm{kg} / \mathrm{dia}$ & 4,06 & 4,37 & 4,06 & 4,38 \\
& \% indicador & 2,7 & 2,5 & 2,8 & 2,6 \\
& $\mathrm{~g} / \mathrm{dia}$ & 110 & 110 & 113 & 113 \\
& & & & & \\
& \% indicador & 7,9 & 7,3 & 9,9 & 9,2 \\
& $\mathrm{~g} /$ dia & 319 & 319 & 402 & 402 \\
Óxido crômico & \% indicador & 0,5 & 0,5 & 0,5 & 0,5 \\
& LDA: lignina em detergente ácido & & & &
\end{tabular}

Os cálculos dos coeficientes de digestibilidade aparente da matéria orgânica obtidos pela técnica de indicadores foram realizados de acordo com Church (1988): Digestibilidade $=100-100 \mathrm{x}$ (A/B), em que:

$\mathrm{A}=\%$ de indicador na dieta ingerida e $\mathrm{B}=\%$ de indicador nas fezes.

As equações para o cálculo de nutrientes digestíveis totais (NDT) e extrativos não nitrogenados (ENN) e a predição da energia digestível (ED) da dieta foram utilizadas conforme Pagan (1998):

$\mathrm{NDT} \%=\mathrm{PD} \%+\mathrm{EED} \% \mathrm{X}(2,25)+\mathrm{FDND} \%+$ ENND\%,

$\mathrm{ENN} \%=100-\mathrm{PB} \%-\mathrm{EE} \%-\mathrm{FDN} \%-\mathrm{MM} \%$, $\mathrm{ED}(\mathrm{kcal} / \mathrm{kg})=255+3660 \mathrm{x}$ NDT, em que:

PD: proteína digestível, EED: extrato etéreo digestível, FDN: fibra em detergente neutro digestível, PB: proteína bruta e MM: matéria mineral.

A acurácia dos indicadores foi avaliada por meio do viés médio, ou seja, pela diferença entre o valor predito pelo indicador e o valor observado pela coleta total de fezes, sendo o mais acurado aquele que possuir viés médio mais próximo do zero (Kohn et al., 1998). O cálculo do viés médio foi realizado segundo a fórmula:

Viés médio $=\sqrt{\frac{\sum(\text { predito }- \text { observado })^{2}}{n^{o} \text { observações }}}, \mathrm{e}$

A precisão é a variabilidade média da distância entre o valor predito e o observado, ou ainda, a variabilidade dos vieses. Ela pode ser avaliada pela raiz quadrada média do erro de predição
(RQMEP) ou pelo erro residual, cujos cálculos foram:

$$
\begin{aligned}
& \text { RQMEP }=\sqrt{\frac{\sum(\text { predito }- \text { observado })^{2}}{n^{0} \text { observações }}}, e \\
& \text { Erro residual }=\sqrt{\left[\mathrm{RQMEP}^{2}-\left(\text { viés }-{\text { médio } \left.)^{2}\right]}^{2}\right.\right.}
\end{aligned}
$$

Para se comparar a acurácia entre os indicadores, o viés médio foi submetido à análise de variância (teste F), e a comparação entre médias foi realizada pelo teste Tukey. Para a determinação da significância do valor médio do viés, foi utilizado o teste $\mathrm{t}$ para média igual a zero, pelo PROC UNIVARIATE do SAS/1998. O nível de significância utilizado foi de 5\%.

Para comparar a precisão entre os indicadores, os valores do erro residual foram submetidos ao teste de homogeneidade de variâncias (teste Hartley), sendo comparados dois a dois, utilizando-se, para tal, o PROC TTEST do SAS/1998.

A fim de se avaliar o comportamento do erro de predição dos marcadores (vieses) em função da variação dos valores observados de DAMO, foi estimada a regressão entre os vieses e DAMO observado (coleta total de fezes), obtendo-se o viés linear (coeficiente angular), o coeficiente de determinação do modelo $\left(\mathrm{R}^{2}\right)$ e a significância do viés para cada indicador, por meio do procedimento REG do SAS/1998.

A avaliação da robustez de cada indicador foi obtida regredindo-se o viés contra as variáveis selecionadas (extrato etéreo na dieta, energia 
digestível na dieta e peso vivo) também pelo procedimento REG do SAS/1998. A comparação entre os coeficientes angulares das retas foi realizada pela metodologia de comparação de retas, cujo princípio é avaliar a interação entre a variável selecionada e os indicadores, por meio da análise de variância (teste F) pelo PROC GLM do SAS/1998, sendo comparado dois a dois (Meyer, 2003).

Dados de digestibilidade foram submetidos à análise de variância de máxima verossimilhança restrita (REML) para um delineamento em quadrado latino 4 × 4 com parcelas subdivididas, mediante ajuste de um modelo linear com efeitos fixos de período de coleta, animal, processamento da alfafa (rama ou cubo), adição de óleo na dieta, método de determinação da produção fecal de matéria orgânica e interação dois últimos fatores. Para isso, foi utilizado o procedimento Mixed do SAS/1998.

Para a obtenção das médias de digestibilidade aparente da matéria orgânica em função do método de determinação da produção fecal (coleta total e indicadores testados) e da adição ou não de gordura na dieta, foram utilizados procedimentos interativos de quadrados mínimos (LSMEANS), e suas comparações foram realizadas por meio do teste $\mathrm{t}$ com $5 \%$ de significância.

\section{RESULTADOS E DISCUSSÃO}

A estatística descritiva da recuperação fecal dos indicadores internos LDA e cutina e do indicador externo óxido crômico é apresentada na Tab. 2.

Tabela 2. Recuperação fecal dos indicadores cutina, lignina em detergente ácido (LDA) e óxido crômico para estimativa da digestibilidade aparente da matéria orgânica em equinos

\begin{tabular}{cccc}
\hline Parâmetro & Cutina & LDA & Óxido crômico \\
\hline Recuperação fecal & & & 16 \\
n & 12 & 12 & $88 \mathrm{~B}^{* *}$ \\
média, \% & $151 \mathrm{~A} *$ & $100 \mathrm{~B}^{\mathrm{ns}}$ & 98 \\
máxima, \% & 196 & 135 & 73 \\
mínima, \% & 76 & 67 & 6,90 \\
desvio padrão & 33,4 & 20,7 & 7,87 \\
CV & 22,1 & 20,7 &
\end{tabular}

Médias na mesma linha, seguidas de letras distintas, diferem entre si pelo teste HSD-Tukey $(\mathrm{P}<0,05)$.

${ }^{1}$ Probabilidade do teste $\mathrm{t}$ para média $=100, \mathrm{~ns}=$ não significativo $(\mathrm{P}>0,05) ;{ }^{*} \mathrm{P}<0,01 ; * * \mathrm{P}<0,0001$.

$\mathrm{CV}=$ coeficiente de variação.

Houve diferença para a recuperação fecal entre os indicadores utilizados $(\mathrm{P}<0,05)$. A recuperação para a cutina foi maior quando comparada à da LDA e óxido crômico, e não houve diferença entre os dois últimos. Aplicando-se o teste $t$ para média igual a 100, pode-se verificar que apenas para a LDA houve recuperação fecal adequada, ou seja, de 100\%, enquanto as quantidades dos indicadores cutina e óxido crômico recuperadas nas fezes foram maiores e menores que 100 , respectivamente. A alta recuperação fecal da cutina pode ter levado a uma subestimativa da excreção da fração matéria orgânica pelas fezes, o que resultou em coeficientes de digestibilidade, aproximadamente, $10 \%$ maiores. Ao contrário, a recuperação fecal da lignina igual a $100 \%$ permitiu valores estimados da DAMO iguais ao obtido pelo método de coleta total de fezes.

Valores médios de recuperação fecal do óxido crômico de $100 \%$ foram relatados por Almeida et al. (1997) que consideraram esse indicador como o mais adequado para ensaios de digestibilidade em equinos, em comparação com a fibra em detergente ácido (FDA), lignina em detergente ácido (LDA) e cinza insolúvel em detergente ácido (CIDA), diferente dos resultados encontrados no presente trabalho.

Oliveira et al. (2003) concluíram que o óxido crômico foi inadequado para as estimativas de digestibilidade em equinos, pois relacionaram esses resultados com a baixa recuperação do indicador nas fezes. A baixa recuperação fecal do 
óxido crômico em ensaios de digestão com equinos foi relatada por Pereira et al. (1995) e Mauricio et al. (1996), de 82,7 e 80\%, respectivamente, valores menores que os encontrados no presente estudo. Segundo Cuddeford e Hughes (1990), a excreção fecal diária do óxido crômico não é constante, o que provavelmente levou, no presente estudo, a recuperações sempre abaixo de $100 \%$, visto a amplitude de valores entre $98 \%$ e $73 \%$.

O coeficiente de variação dos indicadores LDA e cutina foram mais elevados, $20,7 \%$ e $22,1 \%$, respectivamente, quando comparados ao do óxido crômico, o que pode ser indicativo da inconstância de seus teores nas fezes ou, ainda, de dificuldades nos procedimentos laboratoriais. A última hipótese também pode ser considerada para explicar a alta recuperação da cutina nas fezes. Provavelmente, a técnica de determinação desse indicador utilizada neste trabalho quantificou erroneamente alguma fração do material analisado como sendo cutina, superestimando a concentração desta nas fezes. Vasconcellos et al. (2007) trabalharam com LDA, óxido crômico e CIDA como indicadores em dietas para gatos e obtiveram taxa de recuperação da lignina variável entre as quatro dietas utilizadas e elevado coeficiente de variação. Esse fato foi atribuído à lignina em detergente ácido ser relativamente inespecífica, sofrendo influência de outros compostos presentes no alimento ou nas fezes, que podem ser quantificados como lignina.

Os coeficientes de digestibilidade aparente da matéria orgânica das dietas compostas por alfafa, com adição ou não de óleo de soja, obtidos pelos diferentes métodos, a probabilidade de ocorrer diferenças entre os métodos, assim como de ocorrer interação dieta versus método, encontram-se na Tab. 3.

Tabela 3. Médias dos quadrados mínimos da digestibilidade aparente da matéria orgânica (\%) em função do método de determinação da produção fecal e das dietas utilizadas no experimento

\begin{tabular}{lcccccc}
\hline \multicolumn{1}{c}{ Dieta } & Coleta total & Cutina & LDA & Óxido crômico & \\
\hline & & & & & média & CV \\
\cline { 4 - 6 } Com óleo & 70,2 & 79,1 & 68,6 & 66,5 & 71,1 & 7,45 \\
Sem óleo & 65,9 & 76,7 & 66,3 & 60,3 & 67,3 & 10,9 \\
Média & & & & & \\
CV & $68,1 \mathrm{~B}$ & $77,9 \mathrm{~A}$ & $67,5 \mathrm{~B}$ & $63,4 \mathrm{C}$ & \\
\hline Efeito & 5,67 & 5,31 & 7,20 & 7,67 & $\mathrm{Pr}>\mathrm{F}$ \\
Dieta & & & & $<0,01$ \\
Método & & & & $<0,0001$ \\
Dieta x Método & & & & 0,53 \\
\hline
\end{tabular}

Médias seguidas de letras distintas na linha diferem entre si pelo teste $\mathrm{t}(\mathrm{P}<0,05)$.

LDA= lignina em detergente ácido; $\mathrm{CV}=$ coeficiente de variação.

Não foi objetivo discutir diferenças de digestibilidade entre dietas, mas sim mostrar que houve diferença na estimativa da DAMO da dieta entre os métodos, não ocorrendo interação com os tipos de dietas utilizadas. Isso quer dizer que, independentemente do método utilizado, diferenças entre as dietas teriam sido observadas da mesma forma, sugerindo que, em ensaios em que o objetivo é apenas avaliar diferenças qualitativas na digestibilidade entre dietas, todos os indicadores seriam adequados.

O valor médio obtido neste experimento para a DAMO pelo método da coleta total de fezes, $68,1 \%$, foi semelhante ao obtido pelo indicador
LDA, 67,5\%. Ambos foram menores quando comparados ao estimado pela cutina, $77,9 \%$, e maiores que o obtido com o óxido crômico, $63,4 \%(\mathrm{P}<0,05)$.

Alguns autores sugerem que não se utilize a LDA como indicador interno (Cochran et al., 1988; Tamminga et al., 1989) ou que seu uso seja limitado a dietas que contenham mais de $5 \%$ de lignina na matéria seca. Krysl et al. (1988) e Judkins et al. (1990) recomendaram a LDA como indicador interno em determinados tipos de dieta. Ferret et al. (1999) concluíram que a LDA, entre vários indicadores testados, foi o melhor para 
estimar a digestibilidade aparente de matéria seca em dieta à base de alfafa.

Mauricio et al. (1996) avaliaram dietas mistas com feno de coast cross e concentrado para equinos utilizando a lignina na estimativa do coeficiente de digestibilidade aparente da matéria orgânica e observaram que os coeficientes de digestibilidade de FDN e FDA estimados pela lignina foram mais baixos que os observados ao se utilizar coleta total de fezes.

A composição química da lignina nos alimentos é bastante variável, uma vez que esse polímero é composto por várias unidades de p-cumaril álcool, coniferil álcool e sinapil álcool, que variam amplamente em proporções de acordo com a região anatômica em que a lignina está presente na planta e o estágio de maturidade, e, também, entre os diferentes vegetais. Dessa forma, pode ser normal a grande variedade de resultados na literatura devida, principalmente, à diferença entre dietas, ingredientes e protocolos laboratoriais utilizados nos experimentos (Vasconcellos et al., 2007).

Os valores referentes às medidas de acurácia e precisão dos indicadores são apresentados na Tab. 4.

Tabela 4. Parâmetros de avaliação da acurácia e precisão da estimativa da digestibilidade aparente da matéria orgânica (DAMO) em equinos pelos indicadores cutina, lignina em detergente ácido (LDA) e óxido crômico

\begin{tabular}{|c|c|c|c|}
\hline Parâmetro & Cutina & LDA & Óxido crômico \\
\hline $\mathrm{n}$ & 12 & 12 & 16 \\
\hline DAMO observada & 68,1 & 68,1 & 68,1 \\
\hline DAMO predita & 77,2 & 66,7 & 63,4 \\
\hline Viés médio ${ }^{1,2}$ & $9,97 \mathrm{~A} *$ & $-0,56 \mathrm{~B}^{\mathrm{ns}}$ & $-4,69 \mathrm{~B} * *$ \\
\hline $\mathrm{RQMEP}^{3}$ & 11,7 & 6,07 & 5,40 \\
\hline Erro residual $^{3}$ & 7,19 & 7,02 & 3,14 \\
\hline
\end{tabular}

Regressão linear entre vieses e DAMO observadas

\begin{tabular}{cccc}
\cline { 2 - 3 } Viés linear $^{4}$ & $-1,28 \mathrm{~B}$ & $-1,02 \mathrm{~B}$ & $0,03 \mathrm{~A}$ \\
$\mathrm{R}^{2[5]}$ & 0,6263 & 0,4222 & 0,0017 \\
$\operatorname{Pr}>|\mathrm{t}|^{6}$ & 0,0022 & 0,0222 & 0,8798 \\
\hline
\end{tabular}

${ }^{\mathrm{T}}$ Médias na mesma linha, seguidas de letras distintas, diferem entre si pelo teste HSD-Tukey, $(\mathrm{P}<0,05)$.

${ }^{2}$ Probabilidade do teste $t$ para média $=0, \mathrm{~ns}=$ não significativo $(\mathrm{P}>0,05) ; * \mathrm{P}<0,01 ; * * \mathrm{P}<0,0001$.

${ }^{3} \mathrm{RQMEP}=$ Raiz quadrada da média do erro de predição. Valores na mesma linha não diferem entre si pelo teste de Hartley $(\mathrm{P}<0,05)$.

${ }^{4}$ Coeficiente angular do modelo de regressão entre vieses e DAMO. Valores na mesma linha, seguidos por letras distintas, diferem entre si pela metodologia de comparação de retas (análise de variância, interação método x DAMO $-\mathrm{P}<0,05)$.

${ }^{5}$ Coeficiente de determinação do modelo linear de regressão vieses = DAMO.

${ }^{6}$ Probabilidade de aceitação da hipótese nula: viés linear $=0$.

Ao analisar os vieses médios, nota-se que o indicador cutina estimou a DAMO com maior viés $(9,97)$, seguido pelo óxido crômico $(-4,69)$ e pela LDA $(-0,56)$. Os vieses médios do óxido cromo e da LDA não diferiram $(\mathrm{P}>0,05)$, entretanto apenas o viés médio da LDA foi considerado igual a zero pelo teste $t$, mostrando que a lignina em detergente ácido foi o indicador mais acurado para a estimativa da DAMO.

Quanto à precisão, o óxido crômico apresentou raiz quadrada do erro de predição (RQMEP) e erro residual numericamente menores $(5,40$ e
3,14 , respectivamente) que a cutina $(11,7$ e 7,19$)$ e a LDA $(6,07$ e 7,02), sugerindo ser o indicador mais preciso, apesar de não ter havido diferença estatística entre os valores $(\mathrm{P}>0,05)$.

Ao se regredir viés (DAMO predita - DAMO observada) versus (DAMO observada), verificam-se vieses lineares (coeficientes angulares das retas) de $-1,28$ e $-1,02$ para os indicadores cutina e LDA, respectivamente, e de 0,03 para óxido crômico. O viés linear do indicador óxido crômico diferiu dos vieses dos indicadores cutina e LDA, os quais não foram 
diferentes entre si $(\mathrm{P}>0,05)$. Os vieses lineares da cutina e da LDA foram diferentes de zero $(\mathrm{P}<0,05)$, o que não ocorreu para o óxido crômico. Isso significa que o viés, ou o erro de predição, dos indicadores cutina e LDA é influenciado pelo DAMO da dieta. Para cutina e LDA, o viés é maior para predição de DAMO observada, com valores em torno de $60 \%$, e é menor, aproximando-se de zero, quando esses valores estão perto de $70 \%$. Para o óxido crômico, o viés mantém-se constante, independente da DAMO observada.
Dificuldades foram encontradas para comparar estes resultados com os poucos estudos até então publicados, e que avaliaram a cutina como indicador para estimar digestibilidade de frações de dietas para animais.

$\mathrm{Na}$ Tab. 5, é apresentada a avaliação da robustez (coeficiente angular, $\mathrm{R}^{2}$ e probabilidade) das estimativas da DAMO em equinos, por meio dos indicadores cutina, LDA e óxido crômico.

Tabela 5. Avaliação da robustez (coeficiente angular, $\mathrm{R}^{2} \mathrm{e}$ probabilidade) das estimativas da digestibilidade aparente da matéria orgânica em equinos, por meio dos indicadores cutina, lignina em detergente ácido (LDA) e óxido crômico

\begin{tabular}{llll}
\hline Parâmetro & Cutina & LDA & Óxido Crômico \\
\hline
\end{tabular}

EE na dieta, \%

$\begin{array}{lccc}\text { Coeficiente angular } & 1 & -0,37486 & 0,2638 \\ \mathrm{R}^{2[2]} & -0,31665 & 0,0430 & 0,1192 \\ \operatorname{Pr}>|\mathrm{t}|^{3} & 0,0294 & 0,5177 & 0,1902\end{array}$

ED na dieta, Mcal/kg

$\begin{array}{lccc}\text { Coeficiente angular } & -11,9548 \mathrm{~B} & -5,98039 \mathrm{AB} & 4,2729 \mathrm{~A} \\ \mathrm{R}^{2} & 0,2397 & 0,0627 & 0,1659 \\ \operatorname{Pr}>|\mathrm{t}| & 0,1062 & 0,4325 & 0,1174\end{array}$

Peso vivo animal,kg

\begin{tabular}{lccc} 
Coeficiente angular & 0,0665 & 0,1395 & 0,01725 \\
$\mathrm{R}^{2}$ & 0,0204 & 0,0696 & 0,0093 \\
$\operatorname{Pr}>|\mathrm{t}|$ & 0,6577 & 0,4074 & 0,7228 \\
\hline
\end{tabular}

$\mathrm{EE}=$ Teor de extrato etéreo dietético, com base na matéria seca; $\mathrm{ED}=$ Teor de energia digestível na dieta, com base na matéria seca.

${ }^{1}$ Estimativa do coeficiente angular da equação linear de regressão entre os vieses e a respectiva variável independente. Valores na mesma linha foram comparados pela metodologia de comparação da inclinação de retas (análise de variância, interação método x variável independente, $\mathrm{P}<0,05$ ) e quando seguidos de letras distintas, diferem entre si.

${ }^{2}$ Coeficiente de determinação do modelo de regressão linear entre vieses e a variável independente estudada.

${ }^{3}$ Probabilidade de aceitação da hipótese nula: coeficiente angular $=0$.

A robustez dos indicadores foi avaliada por meio do comportamento dos vieses em função das varáveis de interesse. Não houve diferença $(\mathrm{P}>0,05)$ entre os coeficientes angulares da regressão dos vieses dos indicadores estudados e as variáveis extrato etéreo na dieta e peso vivo animal; não diferiram de zero pelo teste $t$ $(\mathrm{P}>0,05)$. Tal fato mostra que todos os indicadores foram robustos quanto à variação do EE na dieta e no peso vivo dos animais utilizados, mantendo sua capacidade de predição da DAMO frente à amplitude de valores das variáveis independentes de interesse.

O mesmo ocorreu para a robustez dos indicadores quanto à energia digestível da dieta, independentemente de os coeficientes angulares da regressão terem diferido entre si $(\mathrm{P}<0,05)$, com o óxido crômico apresentando maior valor, seguido por LDA e cutina. Dessa forma, pode-se dizer que os indicadores cutina, LDA e óxido crômico mostraram-se robustos para todas as 
variáveis testadas (EE, ED na dieta e peso vivo), uma vez que não sofreram influência delas $(\mathrm{P}>0,05)$.

Poucos estudos realizaram o mesmo tipo de abordagem estatística para a avaliação de indicadores, podendo-se destacar o trabalho de Stein et al. (2006). Esses autores observaram que os indicadores utilizados para a estimativa da digestibilidade da matéria seca em equinos foram robustos quanto a variações na dieta $\mathrm{e}$ características dos animais utilizados (peso vivo e idade). Esses resultados foram confirmados no presente trabalho. Verificaram, ainda, que os indicadores utilizados (FDAi, celulose indigestível e cinzas indigestíveis em detergente ácido) apresentaram viés linear significativo $(\mathrm{P}<0,05)$, confirmando os achados deste trabalho para cutina e lignina.

\section{CONCLUSÕES}

Segundo o modelo de avaliação utilizado, conclui-se que o indicador interno cutina e o indicador externo óxido crômico não se mostraram eficientes para estimar a digestibilidade aparente da matéria orgânica nas dietas para equinos compostas por concentrado, alfafa em rama, alfafa em cubos e óleo de soja. Como a cutina tem sido testada como indicador interno em equinos, recomenda-se que novos estudos sejam realizados, com outros tipos de dietas, com a finalidade de validar seu uso nessa espécie animal. $\mathrm{O}$ indicador interno lignina em detergente ácido apresentou recuperação fecal média de $100 \%$ e maior acurácia, desse modo, pode ser considerado o mais adequado para estimar a digestibilidade aparente da matéria orgânica.

\section{REFERÊNCIAS BILBLIOGRÁFICAS}

ALMEIDA, F.Q.; VALADARES FILHO, S.C.; LEÃO, M.I. et al. Digestão pré-cecal em equinos: 2 - Estimativa do fluxo de digesta ileal com indicadores externo e internos. In: REUNIÃO ANUAL DA SOCIEDADE BRASILEIRA DE ZOOTECNIA, 34., 1997, Juiz de Fora. Anais.... Juiz de Fora: SBZ, 1997. v.4. p.178-180.

ARAÚJO, K.V.; LIMA, J.A.F.; FIALHO, E.T. et al. Avaliação de períodos de coleta total de fezes para determinar a digestibilidade aparente dos nutrientes em equinos. Ciênc. Agrot., v.27, p.886-893, 2003.

CHURCH, D.C. The ruminant animal digestive physiology and nutrition. Englewood Cliffs: $\mathrm{O} \&$ Books, 1988. 564p.

COCHRAN, R.C.; VANZANT, E.S.; DELCURTO, T. Evaluation of internal markers isolated by alkaline hydrogen peroxide incubation and acid detergent lignin extraction. $J$. Anim. Sci., v.66, p.3245-3251, 1988.

CUDDERFORD, D.; HUGUIES, D.A. comparison between chromium-mordant hay and acid insoluble ash to determine apparent digestibility of a chaffed, molassed hay/straw mixture. Equine Vet. J., v.22, p.122-125, 1990.

FERRET, A.; PLAIXATS, J.; CAJA, G. et al. Using markers to estimate apparent dry matter digestibility, faecal output and dry matter intake in dairy ewes fed Italian ryegrass hay or alfafa hay. Small Rumin. Res., v.33, p.145-152, 1999.

JUDKINS, M.B.; KRYSL, L.J.; BARTON, R.K. Estimating diet digestibility: a comparison of 11 techniques across six different diets fed to rams. J. Anim. Sci., v.68, p.1405-1415, 1990.

KOHN, R.A.; KALSCHEUR, K.F.; HANIGAN, M. Evaluation of models for balancing the protein requirements of dairy cows. J. Dairy Sci., v.81, p.3401-3414, 1998.

KRYSL, L.J.; GALYEAN, M.L.; ESTELL, R.E. et al. Estimating digestibility and faecal output in lambs using internal and external markers. $J$. Agric. Sci., v.111, p.19-25, 1988.

LIPPKE, H.; ELLIS, W.C.; JACOBS, B.F. Recovery of indigestible fiber from feces of sheep and cattle on forage diets. J. Dairy Sci., v.69, p.403-412, 1986.

MAURICIO, R.M.; GONCALVES, L.C.; RESENDE, A.C. et al. Determinação da digestibilidade aparente em equídeos através do óxido crômico, da lignina e da coleta total de fezes. Arq. Bras. Med. Vet. Zootec., v.48, p.703$711,1996$.

MEYER, P.M. Fatores não nutricionais que afetam as concentrações de nitrogênio uréico no leite. 2003. 131f. Tese (Doutorado em Agronomia) - Universidade de São Paulo, Escola Superior de Agricultura Luiz de Queiroz, Piracicaba, SP. 
NUTRIENT requirements of horses. 5.ed. Washington, DC: Nacional Academy of Science, 1989. 100p.

OLIVEIRA, C.A.A.; ALMEIDA, F.Q.; VALADARES FILHO, S.C. et al. Estimativa da digestibilidade aparente de nutrientes em dietas para equinos, com o uso de óxido crômico e indicadores internos. Rev. Bras. Zootec., v.32, p.1681-1689, 2003.

PAGAN, J. Forages for horses: More than just a filler. In: PAGAN, J.D., GEOR, R.J. (Eds.). Advances in equine nutrition. Manor Farm, Thrumpton, UK: Nottingham University, 1998. p.13-27.

PEREIRA, J.C.; QUEIROZ, A.C., CARMO, M.B. Avaliação de métodos para determinação da digestibilidade aparente em equinos. Rev. Soc. Bras. Zootec., v.24, p.382-390, 1995.

SILVA, D.J. Análise de alimentos (métodos químicos e biológicos). Viçosa: UFV, 1990. $1665 \mathrm{p}$.

SILVA, L.D.F.; EZEQUIEL, J.M.B.; AZEVEDO, P.S. et al. Uso da cutina na estimativa das digestões total e parcial de alguns componentes de rações contendo diferentes fontes de nitrogênio, em bovinos. Rev. Bras. Zootec., v.35, p.600-606, 2006.

STEIN, R.B.S.; TOLEDO, L.R.A.; ALMEIDA, F.Q. et al. Estimativa da digestibilidade aparente da matéria seca por meio de indicadores internos em equinos. Rev. Bras. Zootec., v.35, p.504-511, 2006.

TAMMINGA, S.; ROBINSON, P.H.; MEJILS, $\mathrm{S}$. et al. Feed components as internal markers in digestion studies with dairy cows. Anim. Feed Sci. Technol., v.27, p.49-57, 1989.

UDEN, P.; COLUCCI, P.E.; VAN SOEST P.J. Investigation of chromium, cerum and cobalt as markers in digesta. Rate of passage studies. $J$. Agric. Sci., v.31, p.625-632, 1980.

VAN SOEST, P.J. Nutritional ecology of the ruminant. Ithaca: Comstock Publishing Associates/Cornell University, 1994. 476p.

VASCONCELLOS, R.S.; CARCIOFFI, A.C.; OLIVEIRA, L.D. et al. Utilização de indicadores para estimar a digestibilidade aparente em gatos. Arq. Bras. Med. Vet. Zootec., v.59, p.466-472, 2007. 